\title{
III. Memoir on the best method of decomposing the chromate of iron, obtaining oxide of chrome, preparing chromic acid, and on some combinations of the latter
}

\section{Vauquelin}

To cite this article: M. Vauquelin (1810) III. Memoir on the best method of decomposing the chromate of iron, obtaining oxide of chrome, preparing chromic acid, and on some combinations of the latter , Philosophical Magazine Series 1, 35:141, 20-31, DOI: $10.1080 / 14786441008563012$

To link to this article: http://dx.doi.org/10.1080/14786441008563012

$$
\text { 曲 Published online: } 18 \text { May } 2009 .
$$

Submit your article to this journal ए]

\section{山 Article views: 2}

Q View related articles $\longleftarrow$ 
table food, and with the drink of fermented liquor; and it is as little likely to be destroyed, as the muriate of soda also induced in the very same way. But our foc.' and drink do not, commonly at least, contain the soda united to a destructible acid, or an oxide.

" 9. It is plain, from the preceding, experimesits, that expectorated matter belongs to the class of coagulable fluids, and not of gelatinizable, or, as commonly asserted, mucous fluids. It differs from the coagulable fluid, serum of blood, in forming a much thicker fluid with a much larger proportion of water : for serum, and also the water of blisters, is quite liquid, although they afford, on exsiccation, one-twelfth to one-eleventh of their weight of brittle residue, while some kinds of expectorated matter, of the consistence of mucilage, afford only one-fortieth of dry residue, and others of the consistence of thin paste afford only onefourteenth of residue.

" 10. But for the unavoidable extent of this paper, I should trouble the learned Society with various other conclusions and remarks, especially concerning the glnlularity of expectorated matter, which seems to indicate organization. Although Antonius Van Lewenhoeck, above a century ago, discovered the globularity of the blood, and even noticed it in other animal fluids, neither be, nor any other person, as far as I know, investigated the subject in any fluid but the-blood, till by $\mathrm{Mr}$. Home's acuteness and industry, at a very early period of life, it was observed in pus. I have in this paper related, that expectorated matter, especially the opaque ropy kind, as well as the puriforma is full of globules, and that, except by such agents as destroy charcoal, they are scarcely destructible. Do these spherical particles consist chiefly of organized carbonaceous matter ?"

III. Memoir on the lest Method of decomposing the Chromate of Iron, obtaining Oxide of Chrome, preparing Chramic Acid, and on some Combinations of the latter. By M. Vauquelin *.

$\mathbf{W}_{\text {Hex I made my first experiments on chrome, I had }}$ such a small quantity at my command that it was impossible to vary them so as to bring all its properties before my view.

- From Annale de Chimie, tome lxx. p. 70. 
The importance, however, of the oxide of chrome, on account of its beauty and solidity as a green pigment for earthenware, and in forming imitations of emerald, added to the discovery of large quantities of chromate in the department of the Var, determined me to resume the subject, ard to study the properties of chrome at greater length. In the experiments about to be detailed I was assisted by M. Robiquet, an eminent chemist of Paris.

\section{Process for decomposing the Chromate of Iron.}

Chromate of iron is generally employed in order to procure a large quantity of the oxide of chrome: this ore has for its matrix a kind of steatite, which, from its colour and some other physical properties, might be confounded to a certain extent with chromate itself, the more easily because these two substances at first sight seem to form one and the same mass : after a little attention, however, we find that the matrix is composed of long and pearl-like laminx, whereas chromate of iron is very fine grained, shining, and denser than the matrix.

I had formerly employed, in preparing the oxide of chrome, three parts of nitre to one of the ore reduced to fine powder; but I have since found this proportion was by far too large: indeed, as we can isolate the matrix but very imperfectly, it happens that the nitre, by means of the alkali which it sets free, attacks not only the chromate of iron, but also the alumine and silex, which are there in large proportions; and thus the chromate of potash is mixed with an alkaline solution of all these earths, from which two inconveniences result : in the first place, we are under the necessity of employing, in the separation of these earths and the saturation of the excess of alkali, a great quantity of nitric acid, and if it happens that we go beyond the quantity of acid necessary to the exact saturation of the potash, we redissolve a portion of silex, but principally of alumine : in the second place, these earths, on being precipitated, carry down with them chromate of potash, which cannot be freed from them by washing: a third inconvenience occurs, particularly when we operate on a large scale, and when the heat is -necessarily long continued, -this excess of alkali attacks the crucible and melts it. Thus the œconomy and success of the operation require that we should employ only one half part of nitre to one of chromate : by these means the mass does not enter into fusion, and the chromate is well attacked. It has often happened 
that the potash has been entirely saturated with chromic acid.

This decomposition being effected, the mass is to be well lixiviated, the residue is then to be treated, when hot, with muriatic acid diluted in water, which takes up the iron, magnesia, alumine, and silex, divided by the action of the potash and the subtraction of the chromic acid.

The solution being terminated, the acid liquor is speedily decanted, otherwise it would go into a jelly, and it would then be very difficult to separate the undecomposed chromate : the latter is to be once more treated as at first ; but instead of employing the same quantity of nitre, a fourth part will be sufficient. When the chromate of iron is entirely decomposed, we mix the solutions of alkaline chromate, in order to saturate them by the nitric acid, after which it is proper to crystallize this chromate, as well in order to separate some portions of earth which would have been dissolved by the excess of acid, as to take up a little chromate of iron, which is separated in brown dust by the progress of evaporation. We redissolve in water, filter, and precipitate the liquor by a solution of nitrate of mercury at the minimum, containing the least possible quantity of acid in excess.

Even supposing that the chromate of potash has been purified as we have indicated, $i$. e. that it contains neither earthy substance nor muriate, the chromate of mercury is precipitated in a more or less intense colour, according to the state of concentration of the solutions, their temperature, and excess of acid. In some circumstances the molecules of this salt, by approaching each other more slowly, assume more aggregation, even crystallize, and thereby acquire a deeper red colour. We may also remark that the first portions precipitated are the palest, because, in proportion as we subtract chromic acid, it has the same effect on chromate of potash as if we diluted the liquor. To conclude, the colour has no influence on the quality of the chromate of mercury.

When the mercurial solution is employed at the minimum of oxidation, and as neutral as possible, there remains nothing in the mother water except nitrate of potasb and nitrate of mercury when a superabundance of these has been introduced: but in general these mother waters retain an amethyst colour, and yield with the alkalis a pale green precipitate, which, when heated, leaves oxide of chrome. 
I have attentively examined this precipitate, in order to become better acquainted with its nature and properties.

When treated cold with caustic alkali it dissolves, and communicates a fine green colour to the liquor : a red powder remained which presented all the characters of oxide of mercury at the maximum. The alkaline solution, being filtered and subjected to ebullition, deposited a great quantity of green flakes of oxide of chrome, and prestrved a fine golden yellow colour: this was chromate of potash.

Having diluted in water a portinn of the precipitate obtained by the alkali of the above mother waters; I remarked that it was composed of two different substances; the first was flaky and light; the second, which always occupied the bottom of the mixture, was formed of small crystals of a violet brown. These last presented the following properties: 1st, Wben thrown on burning charcoal, they are entirely volatilized and condensed in small purple needles on such cold bodies as are exposed to their fumes. 2d, When heated more slowly in a retort, they furnish mercury, and leave green oxide of chrome as a residue : they are dissolved in weak nitric acid, communicating to it a fine yellow colour: if we pour into this solution nitrate of mercury at the minimum, common chromate of mercury is precipitated. $3 d$, When we treat this substance by a caustic alkali, the latter acquires a yellow colour, and a red powder remains which is oxide of mercury at the maximum, while the common chromate of mercury' gives black oxide by the same process.

The greenish precipitate obtained by the saturation of the mother waters, by means of an alkali, contains therefore green oxide and chromate of mercury at the maximum.

From what is above stated, we may easily explain what takes place when the motber waters, although containing mercury in excess, nevertheless give a precipitate of chromate of mercury by the addition of fresh nitrate. The reason of this is, that although the nitrate of mercury at the maximum precipitates the chromate of potash, it requires but a very small quantity of acid in order to be dissolved : this in clined me to think formerly that this precipitation had not taken place, because the mercurial solution, containing always an excess of acid, and being rarely at the perfest minimum, the chromate at the maximum, on account of an excess of acid, is kept in solution; but when we add a fresh quantity of nitrate of mercury, the portion at the minimum takes off the chromic acid from the red oxide which is deposited : if B 4 on 
on the contrary we add alkali, we precipitate this chromate at the maximum.

It now remains to explain how oxide of chrome is found in this precipitate:- the following experiment seems adapted to give a demonstration of it. When we treat chromate of mercury at the minimum, by the nitric acid, the solution is effected without the extrication of nitrous gas; but if we reduce the quantity of alkali necessary for the saturation of the acid, we obtain in the first place a brownish-red sediment formed of chromate of mercury at the maximum : the solution by this subtraction becomes green, and precipitates, on the addition of a fresh quantity of alkali, green oxide of chrome, which is easily rodissolved in an excess of caustic alkaii. In this case, to all appearance, a portion of the chromic acid is de-oxygenated in order to hyperoxidate the mercury, from which result chromate at the maximum and oxide of chrome.

It is therefore certain that the chromate of mercury at the maximum, found in the mother water, may arise from two causes : either it results from the mercurial solution, if it contains oxide at the maximum, or it proceeds from the solution of the nitrate at the minimum, on account of the excess of acid, and then it is found mixed with oxide of chrome.

The solubility of the oxide of chrome in the alkali furnishes the explanation of what passes, when on lixiviating cold, the product of the decomposition of the chromate of iron by nitre, we obtain a green liquor which becomes yellow on ebullition; this is because the green oxide is deposited, which lays us under the necessity of filtrating these lixiviums before heating them, in order to separate this oxide in the state of purity. This last phænomenon inclines us to think, with M. Godon de St.Memin, that the chromate of iron, so called in commerce, contains chrome in the state of oxide; for it is improbable that the chromic acid is reduced at the same time with the nitre. What still confirms the opinion of M. Godon is, that the acids extract a green oxide only from the chromate of iron.

To return to the subject. We shall observe that it is essential to wash the chromate of mercury with a good deal of water, in order to free it completely from the nitrate of putash, which, by the calcination of mercurial chromate, would again form chromate of potash, which produces a commencement of fusion in the oxide of chrome, consequently gives it a deeper shade, and renders it heavier, which 
we may avoid when the chromate of mercury is prepared with proper care.

It is sufficient, in order to obtain the oxide of chrome very pure and of a very fine colour, to heat strongly in a well luted earthen retort the pure chromate of mercury, until no more oxygen is extricated, and to keep up the fire so much the longer in proportion to the depth of the shade we wish to obtain: it seems that there really exist two kinds of oxide of chrome, for by heating it a very long time the green is so weakened, that it passes to a deadleaf yellow.

\section{Combination of the Chromic Acid with Barytes.}

In order to prepare the chromate of barytes, we employ with success chromate of potash well purified and very neutral; we mix with it nitrate of barytes until no more precipitate is produced: we must collect the latter, decant the liquor, and wash several times, until it is entirely freed from all extraneous saline particles.

No harm is done by employing great quantities of water, even warm, in order to wash the salt, for it is not very soluble.

\section{Analysis of the Chromate of Baryles.}

Five grammes of this salt, dissolved in the nitric acid and precipitated by sulphuric acid, gave four grammes and four tenths of sulphate of barytes. Admitting with $\mathbf{M}^{3}$. Klaproth 68 parts of barytes in 100 parts of sulphate, we shall have, in 100 parts of chromate of barytes, $62 \cdot 2$ of base, and $37 \cdot 4$ of acid.

According to this account, the five grammes of chromate of barytes contain one gramme 87 centiemes of concrete acid, and the latter converted to the state of green uxide by a strong calcination, is reduced to one gramme 56 centiemes, which gives a difference between the quantity of oxygen contained in 100 parts of oxide and 100 parts of acid of $16 \cdot 6, i . e$. in order to convert 100 parts of oxide of chrome into acid, we must combine with it $16^{\circ} 6$ of oxygen.

In order to effect the analysis of the chromate of barytes, we must dissolve it in weak nitric acid, aided. by a little heat, and pour into the solution sulphuric acid in excess; we then wash the sulphate of barytes, and weigh it after having dried and calcined it.

With the same view, we may gently evaporate the liquor to dryness, in order to drive off the nitric acid; redissolve the residue in water, saturate it with ammonia, 
and calcine strongly in order to evaporate the sulphate of ammonia and completely decompose the chromate of ammonia : lastly, weigh the oxide of chrome. The latter, when boiled with nitric acid, ought to give no sign of sulphuric acid with the nitrate of barytes.

If the proportions of the chromate of barytes which we have obtained are very exact, as there is every reason to believe they are, the same analysis may serve for those of other chromates which are soluble in water.

\section{Process for obtaining the Chromic Acid pure.}

Among the various methods which may be employed for preparing this acid, the most preferable, in our opinion, consists in decomposing the chromate of barytes by the sul. phuric acid : all other processes were attended with more or less difficulties.

We must therefore dissolve the chromate of barytes in weak nitrous acid; afterwards carefully precipitate it by means of sulphuric acid, so that all the salt may be decompos:d without the sulphuric acid being in excess. If by chance we exceed this point, we must separate the superabundance of the acid by barytes water. We shall then find whether we have seized the point at which the precipitate formed by chromic acid in barytes water is redissolved entirely in nitric acid, and at which the sulphuric acid does not disturb this chromic acid.

We then filter the liquor and carefully evaporate it, particularly towards the latter part of the operation, that we may not decompose the chromic acid: this evaporation must be repeated several times to dryness, in order to expel all the nitric acid.

When the chromic acid is highly concentrated, irregular (mammelonnées) masses are formed, in which we see small red crystals grouped together, but they are not permanent in the air, as they attract humidity from it.

The chromic acid thus purified is of a deep red colour, has a very acid, but austere and metallic taste; it is soluble in alculiol, which speedily decomposes it, for the solution becomes green.

\section{Easy Method of determining the Quantity of Chromic Acid combined or mixed with several Saline Substances.}

We must put into the solution containing chromic acid a slight excess of nitric acid, if the salt be not of itself soluble nor acid: we must then pour in a little hydro-sulphuret of ammonia, and macerate for some time in a close flask, 
after which we must boil in order to drive off the superabundant sulphuretted bydrogen, filter in order to separate the sulphur, pour into the filtered liquor some drops of caustic potash, and thus obtain the green oxide of chrome; filter again, wash carefully, dry and calcine carefully. On adding to the quantity of oxide obtained the 16.6 centiemes of its weight, we have the quantity of chromic acid which any given salt contained. We may easily see that in this operation the sulphuretted hydrogen makes the chromic acid pass to the state of green oxide, which the excess of acid takes up again in proportion as it is precipitated: if some portions of it remain, we succeed in separating it from the sulphur by ebullition, which serves at the same time to drive the sulphuretted hydrogen. The potash afterwards added only serves to decompose the salt of chrome which is formed.

\section{Action of Sulphurous Acid on Chromic Acid.}

If into chromic acid we pour a great quantity of sulphurous acid, its yellowish-red colour passes to a dirty brown; and if at this period we pour caustic alkali into the liquor, we obtain a reddish-brown precipitate, which is dissolved in the acids.

A greater quantity of sulphurcus acid immediately changes the red colour of the chromic acid to a pale green. Thus it should seem that two kinds of oxide of chrome exist, but which scarcely differ in the quantity of oxygen*.

\section{Action' of the Acids on Oxide of Chrome.}

In general the oxide of chrome obtained by the calcination of the chromate of mercury is attacked by the acids with very great difficulty : we at length succeed, however, in dissolving it; but in order to form combinations we made use of the oxide obtained from the decomposition of the chromate of potash by means of the bydro-sulphuret, according to the process above described. We have seen that this oxide, when recently precipitated, is dissolved with the greatest facility even in the weakest acids.

The sulphate of chrome presented nothing renarkable; we merely observed that it is easily decomposed by beat; for, when slightly calcined, it is no longer redissolved in water.

- We obtain an oxide similar to that which is produced by sulphurous acid in the former case, by passing oxygenized muriatic acid into an acid solution of green oxide of chrome, and into which we gradually pour a little potash, 
The muriate is so far remarkable, that when evaporated to dryness, it gives a red powder which attracts humidity from the air; its solution is of a fine green colour. If we calcine it :ather strongly, it gives out a smell of oxygenized muriatic acid, acquires a great bulk, and is transferred into small micaceous brilliant yellow fibres: finally, if we heat it still more, it is tolally converted into green oxide.

We boiled several times, and in large quantities, nitric acid over oxide of chrome recently precipitated, and it was completely dissolved; but when we separated the oxide by means of caustic potash, the supernatant liquor was colourless : the contrary is the case when we evaporate to dryness and slightly calcine: by redissolving in water, the latter assumes a reddish colour; and after the separation of the oxide, the liquor remains of a fine golden yellow.

We also dissolved oxide of chrome in phosphoric and in oxalic acids : the first combination was of a splendid emerald green colour, and the other, when viewed in the mass, presented an amethyst hue. Sulphurous acid also dissolves oxide of chrome with great facility.

\section{Action of the Caustic Alkalis on Oxide of Chrome.}

If into a solution of chrome a little diluted we pour caustic potash in a quantity above what is necessary for the saturation of the acid, the oxide is redissolved in this alkali. We also obtain an alkaline solution of oxide of chrome on taking it recently precipitated, diluting it with a little water, and dissolving in this water some pieces of caustic potash; on afterwards diluting the combination with water and filtering, we obtain a liquor of a fine green, which, on ebullition, deposits the oxide it contains, and the liquor remains colourless.

\section{Chromate of Potash.}

There are two kinds of chromate of potash; the one neutral is of a citron yellow, and crystallizes in small prisms. This salt, on the addition of heat, assumes a fine red, hut returus to its natural colour when cooled. The second has an excess of acid; its colour is orange-red, and it crystallizes in beautiful prisms of the same colour.

\section{Chromate of Ammonia.}

When we saturate ammonia by the chromic acid, and abandon the liquor to a spontaneous evaporation, an arborescent salt is formed out of the liquor, composed of tufts of fine yellow: sometimes it is presented in the form of 
pearl-like laminæ. To conclude, this salt is easily decomposed by heat; even when it is dissolved, brown flakes are separated, which are oxide of chrome, and which become green by calcination.

\section{Chromate of Lime.}

Chromic acid forms a very soluble salt with lime; its solution furnishes by evaporation silky flakes of a yellowish brown, which are easily dissolved in water: this salt is decomposed by the fixed alkalis.

\section{Chromale of Magnesia.}

Magnesia easily combines with chromic acid: the salt which results from it is very soluble in water; the solution crystallizes in prisms with six faces perfectly transparent, and of a fine topáz yellow: when they are in considerable quantity the colour is orange yellow.

Magnesia is separated from the chromic acid by the fixed caustic alkalis and the alkaline earths.

\section{Metallic Chromates.}

If into a solution of sulphate of iron at the minimum we pour chromate of potash, we obtain a fawn-coloured precipitate, which, when treated with caustic alkali, gives no trace of chromic acid: this precipitale is dissolved with great facility in muriatic acid, from which the alkali separates it completely without the least trace of alkaline chromate remaining. The nitric acid dissulves a part of the precipitate, and assumes a fine green colour: this precipitate therefore is not a chromate of iron, but a mixture or combination of oxide of iron and oxide of chrome, which seems to resemble strongly what is presented to us by nature.

It is evident from the result of this experiment, that the chromic acid has been decomposed by the oxide of iron, which, in passing to the maximum, has reduced the sther to the minimum, or to the state of green oxide. If we wished therefore to form chromate of iron, it would be necessary to employ this last metal saturated with oxygen, in order that it may not be able to act upon that of the chrome.

\section{Chromate of Lead.}

This combination assumes different shades, according to the manner in which it has been prepared.

If the chromate of potash be neutral, we obtain an orange-yellow colour; if it has an excess of acid, the co- 
lour is deep citron yellow: if the alkali on the contrary be predominant, the shade is a reddish yellow, and sometimes a fine deep red : the shades also vary in proportion as we operate in the hot or cold manner.

Chromate of lead made with a solution slightly acid is that which is most in request by painters, and it is in fact the most solid. We may heighten its colour, either by a little alkali, or by precipitating it hot with acetate of lead. In the latter case it should seem that a part of the acetic acid is separated, and that the oxide of lead which it abandons is united to the conmon cbromate and heightens its colour.

We should conceive that the chromates which contain an excess of oxide of lead must be more alterable by sulphurous vapours than those in which this oxide is saturated by the chromic acid.

\section{Chromate of Copper.}

The simplest way of forming chromate of copper is to mix a solution of neutral chromate of potash with a solution of sulphate of copper: a brownish-yellow precipitate is formed, which, when well washed and dried, assumes a bistre brown colour.

\section{Chromate of Silver.}

Chromate of silver is prepared by decomposing nitrate of silver by neutral chromate of potash : a reddish-brown precipitate is produced when the operation is performed with heat, and of a purple-red when done in the cold: lastly, it is of a carmine-red colour if the solution of chromate of potash contains a slight excess of acid : in the latter case the precipitate is not so quickly formed, and is less abundant, but it is crystallized in sinall semitransparent grains.

This salt becomes brown on exposure to light; it is soluble in the nitric acid, and from which the muriatic acid separatc:s the oxide of silver.

\section{Uses to which the Preparations of Clirome are applied.}

This oxide is now generally employed in the porcelain manufactories throughout France. It supports better than any other metal, and without undergoing any alteration, the intense heat employed to prepare hard porcelain: it produces an extremely beautiful green, which bas not yet been obtained with any of the other metals.

A very fine enamel resembling in colour the emerald of Peru is made with the oxide of chrome. Another enamel 
is also made with it, which, when applied upon copper or silver, furnishes a colour precisely similar to that of fine gold, and imitates this bright metal extremely well when applied in thin leaves to other metals : a colour which, I think, cannot be obtained in the same degree of perfection with any other metal.

I shall not dwell upon the different varieties of chromates of lead used in painting: they are already well known to artists, and are in great request on account of their beautiful colours, the facility with which they may be applied, and their great inalterability.

It is very probable that several other metallic chromates would also furnish beautiful colours if they were properly examined by painters.

IV. Observations on loaded and untoaded Barges, Boats, Beams, or flouting Bodies descending with Streams or Currents, and why the heavier End will go foremost. By George OrR, Esq.

I

$\mathbf{I}_{\mathrm{N}}$ addition to what I have already said on this subject, I now submit what follows to the consideration of scientific men : and as my object is an endeavour to attain the truth on so interesting a subject, I am ever ready to admit my errors where I am wrong, but hope that reason and good temper, free from peevishmess and personality, will ever regulate the dincussion of philosophical subyjects.

When bodies of the description alluded to, or in fact any bodies that are specifically lighter than water, float in it, there are two powers always opposed to each other, that is, the specific gravities of the fluid and of the floating body; and in proportion as these differ more or less, in the same proportion will the two bodies oppnse each other : for the less specifically heavy any floating body is, the less power will it possess to contend against the fluid in its endeavours to sink or descend; and of course it will follow that it will float nearer the surface. When water is perfectly at rest, it has found its level, and its surface presents a borizontal plane; or in other words, all its particles press or gravitate towards the centre of the earth in perpendicular and right lines; but on any change from this state of rest taking place, the particles of water are, by the force of gravitation, put in motion, and will endeavour to find their level again, or continte to move on an inclined plane; and all bodies suspended in the fluid, or floating on the surface more or less deep, being subject 